fluid from the jar and insures the permaneincy of the specimen. The distinct advantages of the jar here describerl, over the jars now on the market, are that it insures: (1) permanency of the preparation; (2) neatness and permanency of the label.

The jar here described is manufactured by F. A. Hardy \& Co., Chicago.

Medical Laboratory Building.

\section{A NEW PIPET}

\section{J. E. Huber, M.D., Peoria, Ill.}

Some time ago I had difficulty in removing small quantities of separated serum from a small, narrow centrifuge tube. The usual method of capillary attraction with a fine-bore glass tubing pipet failed, owing to the fact that if I tipped

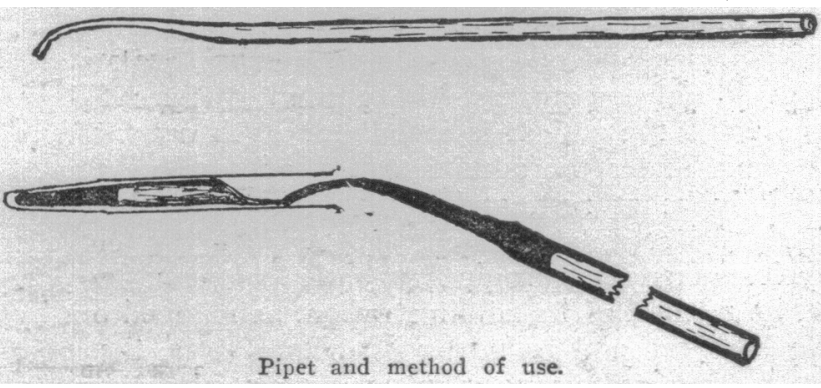

the tube sufficiently for the pipet to act, the serum movement would cause the sediment to mix, defeating the object, which was separation of the serum. In a wide tube it is not difficult to raise the capillary point of the pipet higher than the finger end, high enough to increase the flow materially.

Narrow tubes are much more of a problem. My experience with Wright's pipets for blood collection gave me an idea which I have not seen mentioned. The end of the drawn out part of the pipet, a millimeter in diameter, is carefully flamed so that it curves about 45 degrees, say about one quarter-inch from the tip, as in the accompanying illustration. With such a pipet it is possible to withdraw fluids from watch crystals held on the flat, and from tubes which are too small to permit the use of a straight pipet. It is only necessary to have a clean pipet, and touch the curved end to the liquid, keeping the finger end lower than the curved end. Capillary action will fill the narrow tube beyond the distal curve, and the siphon action does the rest. Its action is instantaneous with aqueous liquids; with serum it is a little slower, but it will drain completely any kind of a tube, straight or curved.

It is simpler than the rubber nipple pipet, and is under better control, as the release of pressure on the nipple sometimes is great enough to cause currents that whirl up the sediment and spoil the experiment.

\section{THE PAINLESS DRESSING}

\section{S. S: Gale, M.D., RoAnoke, VA.}

The dressing is yellow petrolatum with 10 per cent. boric acid incorporated in it. This boric acid ointment is prepared by the druggist and placed in a tin can. The regular petrolatum can of 1 to 5 pounds is an excellent container.

At each dressing the can containing the ointment is placed in a water bath and allowed to melt. This melting also resterilizes the ointment for each dressing. After the ointment has thoroughly melted it is poured into a large, flat, sterile pan. Pieces of gauze of various sizes and thickness, depending on the nature of the wound, are saturated with this melted ointment. After the wound has been sponged off, preferably with alcohol and normal saline, warm dressings are applied directly to the wound surface. These dressings are changed as often as is necessary, cicpending on the nature of the wound. When large areas are to be covered, it is usually best to have rolls of gauze about 8 inches broad and as many thicknesses as are necessary. These rolls are placed in the melted ointment and thoroughly saturated; then they are unfolded and applied directly to the wound. The greasy dressing can then be covered with plain gauze and held in place with a roller bandage.

This dressing does not dam back secretions, nor does it allow the granulations to embed themselves in the meshes of the gauze, and it can be removed absolutely without any pain. It will not dry out for several days. To my mind it is the ideal dressing in a large number of cases in which there is a large raw area to be covered.

\section{A PHARYNGEAL TUBE COMBINING REBREATH- ING AND INSUFFLATION}

Frank Wilcox Pinneo, M.D., Newark, N. J.

In all inhalation anesthesias the most frequent and important embarrassments to respiration are obstructions in the air way. Of all causes of obstruction the tongue falling back on the glottis is altogether the most common. As this fault in technic is within the control of the anesthetist and always removable, means to facilitate holding the tongue forward are practicable without the necessity of holding the jaw forward with the hand. There has been developed in insufflation an improved method over any kind of mask for administering ether, and pharyngeal insufflation has proved that the advantages in almost all kinds of cases may be secured without the necessity of intratracheal apparatus. When the nasal catheters are not used, but introduction by mouth is preferred, the combination of a rebreathing tube, to keep the tongue forward, and a tube for insufflation of the ether is a great convenience, and for this purpose the one shown in the illustration has been devised. It is a one piece metal blade with upturned sides to fit the contour of the tongue, having a channel for introduction of an oral eatheter, 5 inches long; is curved from lips to pharynx, the mouth end being a flattened tube and adjustable for different sized patients. Its advantages are: 1 . It provides for automatic holding of the tongue forward without other means. 2. It has not the objections of a closed tube, being an open blade with channels easily cleaned. 3. It can be used in patients

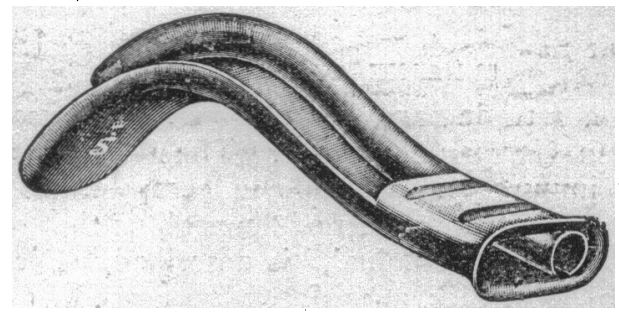

Rebreathing tube combined with a tube for insuffation of ether.

of different sizes. 4. It has a channel for introduction of an oral catheter for pharyngeal insufflation, the catheter not being part of the pharyngeal tube. 5. The anesthetic may therefore be stopped while the blade remains in place. 6. It is efficient in holding the tongue forward without being so cumbersome as a tube like Connell's or a fenestrated frame like Lumbard's. 7. It is efficient for rebreathing, keeping the teeth and lips apart and the tongue forward. 8. It does not get clogged with mucus or blood.

199 Garside Street.

The Returns of Medical Practice.-For a livelihood, the practice of medicine brings in slow return, depending on location and opportunity. Success is laggard, as a rule, and even when emoluments balance expenditures, the profit in the practice of medicine is not considerable. The exceptional physician may grow well to do through fortunate investment, but outside of the larger cities, few get rich.Isadore Dyer, M.Di, New Orleans Med. and Surg. Jour. 